\title{
Introduction: ADSA and Interbull Joint Breeding and Genetics Symposia*
}

\author{
F. Miglior, ${ }^{1} \dagger \oplus$ C. F. Baes, ${ }^{1,2}$ ๑ D. Lourenco, ${ }^{3} \odot$ F. Penagaricano, ${ }^{4} \oplus$ and B. Heins ${ }^{5} \odot$ \\ ${ }^{1}$ Centre for Genetic Improvement of Livestock, Department of Animal Biosciences, University of Guelph, ON, Canada N1G 2W1 \\ ${ }^{2}$ Institute of Genetics, Vetsuisse Faculty, University of Bern, Bern 3001, Switzerland \\ ${ }^{3}$ Department of Animal and Dairy Science, University of Georgia, Athens 30602 \\ ${ }^{4}$ Department of Animal Sciences, University of Florida, Gainesville 32611 \\ ${ }^{5}$ Department of Animal and Science, University of Minnesota, Morris 56267
}

\section{INTRODUCTION}

The Joint ADSA/Interbull Breeding and Genetics Symposia titled "Ten Years of Genomic Selection" and "Data Pipelines for Implementation of Genomic Evaluation of Novel Traits" were held at the 2019 ADSA Annual Meeting, from June 23 to 26, in Cincinnati, Ohio. The objective of the first symposium was to provide a broad overview of 10 years of genomic selection in dairy cattle. In 2009, genomic evaluations were first implemented in the United States and Canada, followed over time by evaluations in many other countries. The rapid uptake of genomic selection has had a dramatic effect on the dairy industry. This symposium highlighted the development and impact of this implementation in all dairy breeds and outlined new developments and future scenarios. The objective of the second symposium was to provide an international view of recent worldwide advances on the development of new data pipelines, with the overall objective of implementing genetic evaluations for novel traits in the era of genomics. The advent of genomics has created an opportunity to focus on and select for expensive traits, which was not feasible with traditional selection. This symposium provided various examples of national and international initiatives to pool data across countries or organizations to exploit the potential of accurate genomic evaluation for novel and expensive traits

Received April 6, 2020.

Accepted April 6, 2020.

*Presented at the Joint ADSA/Interbull Sessions: Breeding and Genetics: Ten Years of Genomic Selection and Data Pipelines for Implementation of Genomic Evaluation of Novel Traits at the ADSA Annual Meeting, Cincinnati, Ohio, June 2019.

†Corresponding author: fmiglior@ontariogenomics.ca

\section{Ten Years of Genomic Selection}

The opening speaker in the first symposium, Derek Bickhart (US Dairy Forage Research Center, Agricultural Research Service, USDA, Madison, WI), presented a talk titled "Advances in sequencing technology herald a new frontier in cattle genomics and genome-enabled selection." This set the stage by reviewing the development of the cattle reference genome and how this was a major inflection point for the start of domestic livestock genomic selection. The enormous effort behind the generation of this resource was well served by the willingness of the assembly consortium to release early data for the development of concurrent marker resources, which facilitated the development of multiple SNP chip panels for fast uptake by the dairy industry for immediate implementation of genomic evaluation and selection (Bickhart et al., 2020).

The second speaker was Paul VanRaden (USDA, Agricultural Research Service, Animal Genomics and Improvement Laboratory, Beltsville, MD), whose presentation was titled "How to implement genomic selection." He reviewed how genomic selection was initially applied to males to predict progeny performance and now has been widely applied to females or even embryos to predict their own later performance. Furthermore, VanRaden illustrated how genomic evaluations are now provided weekly; how predictions from international genomic databases provide more accurate and cost-effective evaluations than from the within-country databases; and how selection indices include many new traits, often with lower heritability or requiring large initial investments to obtain phenotypes, which provide further incentive to cooperate internationally (VanRaden, 2020).

Christian Maltecca (Animal Science Department, North Carolina State University, Raleigh) provided a different perspective in his talk, "Exploiting homozygosity in the era of genomics - Selection, inbreeding, and mating programs." The advent of genomic selection has yielded an unprecedented acceleration of genetic 
progress but has also resulted in rapid accumulation of homozygosity in dairy cattle populations. A better understanding of this homozygosity accumulation will ensure a continuous genetic progress while curtailing the accumulation of deleterious recessives and maintaining enough genetic variance (Maltecca et al., 2020).

The fourth speaker, Esa Mäntysaari (Natural Resources Institute Finland (Luke), Jokioinen, Finland), gave a talk titled "Single-step genomic evaluations in dairy cattle." In his talk, Mäntysaari presented the history of the development of single-step genomic evaluations and its current implementation in various countries. In addition to computing approaches, he reviewed single-step validation results and discussed possible reasons for biases in single-step evaluations (Mäntysaari et al., 2020).

\section{Data Pipelines for Implementation of Genomic Evaluation of Novel Traits}

Rob Tempelman (Department of Animal Science, Michigan State University, East Lansing) was the first speaker in the symposium, and he focused on the topic of "Genetic relationships between different measures of feed efficiency and the implications for dairy cattle selection indices." As selection programs to improve feed efficiency are beginning to be implemented worldwide, Tempelman provided some clarity regarding the relationships between DMI and various feed efficiency traits, including residual feed intake, and, more recently, feed saved, and how those traits should be differentially incorporated into selection indices (Tempelman and $\mathrm{Lu}, 2020$ ).

The second speaker of the symposium, Francesca Malchiodi (Semex Alliance and Centre for Genetic Improvement of Livestock, Department of Animal Biosciences, University of Guelph, ON, Canada), presented the topic of "Multiple trait single-step genomic evaluation for hoof health." Her talk outlined how the joint effort by scientists at the University of Guelph and within the Canadian dairy industry have developed (1) the data pipeline to collect hoof health data from hoof trimmers across Canada; (2) the research to create the basis of a genetic evaluation for hoof health traits and a selection index; and (3) the implementation of the multiple trait single-step genomic evaluation for hoof health in Canada (Malchiodi et al., 2020).

The third speaker, Kristen Parker Gaddis (Council on Dairy Cattle Breeding, Bowie, MD), gave a talk titled "Development, implementation and future perspectives of health evaluations in the United States." Parker Gaddis described how, following a long period of research and development by many, the Council on Dairy Cattle Breeding released national genetic evaluations for resistance to common health events in 2018. Furthermore, she outlined how health evaluations are now included in net merit selection indices, allowing producers to identify animals predicted to be the most resistant to most common diseases (Parker Gaddis et al., 2020).

The fourth speaker, Jennie Pryce (Agriculture Victoria Research, AgriBio, Centre for AgriBioscience, Bundoora, Australia), gave a talk titled "Genomic selection for reducing environmental impact and adapting to climate change." Her talk outlined how the implementation of genomic selection has allowed Australia to focus on traits that can reduce the environmental impact of the dairy industry and improve the adaptability of dairy cows to climate change. In particular, she focused on the implementation of genomic evaluation for heat tolerance released in Australia in 2017. Pryce concluded her talk by describing the worldwide effort to produce genetic evaluations for methane emission, and how genomic selection for methane emission would require international collaboration to assemble a suitably sized reference population (Pryce and HaileMariam, 2020).

These two symposia were developed to provide a comprehensive overview of the last 10 years of genomic selection and how genomics has provided the opportunity to develop new data pipelines for novel traits now under selection worldwide.

\section{REFERENCES}

Bickhart, D. M., J. C. McClure, R. D. Schnabel, B. D. Rosen, J. F. Medrano, and T. P. L. Smith. 2020. Symposium review: Advances in sequencing technology herald a new frontier in cattle genomics and genome-enabled selection. J. Dairy Sci. 103:5278-5290. https: //doi.org/10.3168/jds.2019-17693.

Malchiodi, F., J. Jamrozik, A.-M. Christen, A. Fleming, G. J. Kistemaker, C. Richardson, V. Daniel, D. F. Kelton, F. S. Schenkel, and F. Miglior. 2020. Symposium review: Multiple-trait single-step genomic evaluation for hoof health. J. Dairy Sci. 103:5346-5353. https://doi.org/10.3168/jds.2019-17755.

Maltecca, C., F. Tiezzi, J. B. Cole, and C. Baes. 2020. Symposium review: Exploiting homozygosity in the era of genomics-Selection, inbreeding, and mating programs. J. Dairy Sci. 103:5302-5313. https://doi.org/10.3168/jds.2019-17846.

Mäntysaari, E. A., M. Koivula, and I. Strandén. 2020. Symposium review: Single-step genomic evaluations in dairy cattle. J. Dairy Sci. 103:5314-5326. https://doi.org/10.3168/jds.2019-17754.

Parker Gaddis, K. L., P. M. VanRaden, J. B. Cole, H. D. Norman, E. Nicolazzi, and J. W. Dürr. 2020. Symposium review: Development, implementation, and perspectives of health evaluations in the United States. J. Dairy Sci. 103:5354-5365. https://doi.org/10 .3168/jds.2019-17687.

Pryce, J. E., and M. Haile-Mariam. 2020. Symposium review: Genomic selection for reducing environmental impact and adapting 
to climate change. J. Dairy Sci. 103:5366-5375. https://doi.org/10 3168/jds.2019-17732.

Tempelman, R. J., and Y. Lu. 2020. Symposium review: Genetic relationships between different measures of feed efficiency and the implications for dairy cattle selection indices. J. Dairy Sci. 103:53275345. https://doi.org/10.3168/jds.2019-17781.

VanRaden, P. M. 2020. Symposium review: How to implement genomic selection. J. Dairy Sci. 103:5291-5301. https://doi.org/10 $.3168 /$ jds.2019-17684

\section{ORCIDS}

F. Miglior ๑ https://orcid.org/0000-0003-2345-8842

C. F. Baes ๑ https://orcid.org/0000-0001-6614-8890

D. Lourenco () https://orcid.org/0000-0003-3140-1002

F. Penagaricano @ https://orcid.org/0000-0001-6661-3991

B. Heins (ㄴ) https://orcid.org/0000-0003-2186-9082 